\title{
Medical Errors In U.S. Healthcare Organizations: Have We Made Any Progress?
}

\author{
Dr. Yvette Ghormley, Walden University, USA
}

\begin{abstract}
Since the Institute of Medicine's landmark 1999 report on medical errors, mandates, legislation, and recommendations have been forced on the U.S. healthcare industry. However, only limited progress has been made. Part of the difficulty is identifying the scope of the problem, which has been far larger than thought since the advent of new reporting tools. The major causes of medical errors lie in the lack of a (a) pervasive safety culture, (b) commitment by top healthcare organization management to reduce medical errors, and (c) integrated IT systems, including electronic health records. Compared to other "high reliability" organizations that have achieved excellent results in regard to safety, healthcare is perceived as lagging far behind. Healthcare is not the sole industry needing a safety culture. However, many healthcare leaders perceive existing industry tools that high reliability organizations routinely use as irrelevant. Positive change will come when attitudes change and healthcare organizations embrace the solutions that other industrial organizations have utilized to produce satisfactory safety outcomes.
\end{abstract}

Keywords: Medical Errors; Healthcare Organizations; Electronic Health Records; Quality

\section{INTRODUCTION}

1

n 1999 the Institute of medicine (IOM) reported that at least 44,000 individuals and perhaps as many as 98,000 die in hospitals annually because of medical errors (Institute of Medicine, 1999). The Quality of Health Care in America Committee responsible for conducting the study at the IOM did not suggest this situation was due to recklessness or actions of any particular group but rather that faulty systems, processes, and conditions make such errors much more prone to occur. The report results generated immediate action by the Clinton administration and the U.S. Congress, including instructions to agencies that oversee healthcare programs to implement strategies for reducing medical errors. The action plan at the Agency for Healthcare Research and Quality (AHRQ) comprised developing and testing new technologies, instituting large-scale demonstration projects to test safety interventions and error-reporting strategies, establishing multidisciplinary and geographically diverse research teams, and conducting studies to analyze environmental and educational issues.

Five years after the IOM report, a report from the Commonwealth Fund stated that "notable advances had been made" but considerable barriers remained-for example, how medical errors are quantified (Bleich, 2005). This made the 2004 IOM's goal of a 50\% reduction in errors by 2004 almost irrelevant because the rate was impossible to determine with any accuracy. Further legislation, including the Tax Relief and Health Care Act of 2006, and Quality Assessment and Performance Improvement (QAPI) programs mandated by federal regulation as a condition of Medicare and Medicaid participation then spurred healthcare organizations to report on both adverse events, defined as harm to a patient as a result of medical care, and "never" events, described as serious events that should never occur in a healthcare setting. Consequently, by October 2008, the Office of the Inspector General, Department of Health and Human Services was able to conduct a study that unearthed the following disturbing findings (Department of Health and Human Services, Office of Inspector General, 2010): 
- $\quad$ About $14 \%$ of Medicare beneficiaries experienced an adverse event while in a hospital

- $\quad$ During their hospital stay, an additional $14 \%$ of Medicare beneficiaries experienced events that resulted in temporary harm

- $\quad$ Of these events, physician reviewers determined that $44 \%$ were preventable and $51 \%$ not preventable

- $\quad$ Costs associated with these events totaled $\$ 137$ million, just for the month of October.

In 2009, a new resource- the Global Trigger Tool—became available to further help researchers quantify patient safety (Institute for Healthcare Improvement, 2009). Use of the tool produced startling results. For example, evaluation of three leading hospitals by this tool and two others demonstrated that adverse event detection methods regularly used to track patient safety in the USA - voluntary reporting and the AHRQ's Patient Safety Indicatorsmissed 90\% of adverse events (Classen et al., 2011). In these hospitals one third of all admissions were associated with an adverse event. Most damning of all were the authors conclusions: "Reliance on such methods could produce misleading conclusions about safety in the US health care system and could misdirect patient safety improvement efforts" (Classen et al., 2011, p.6). Employing a weighted average of four studies that utilized the Global Trigger Tool, James (2013) determined that a lower limit of 210,000 deaths per year was associated with preventable harm events in hospitals with the true number of premature deaths at more than 400,000 per year. Perhaps just as staggering was that serious harm occurred at a rate of 10-20 times that of lethal harm.

Clearly, healthcare in the United States has a problem; understanding why the problem is of such a magnitude is the goal of the rest of this review.

\section{WHY HAS PROGRESS BEEN SO LIMITED?}

Is hospital care a special case? Many industries outside of healthcare have demonstrated remarkable safety records based on characteristics and behaviors that create conditions for exemplary performance and "high reliability" (Reason, 1997; Weick \& Sutcliffe, 2007). Therefore, Chassin and Loeb (2013) argued that it might be possible to translate principles operating in high-reliability organizations as defined by Weick and Sutcliffe to healthcare organizations. These principles include preoccupation with failure, reluctance to simplify, sensitivity to operations, commitment to resilience, and deference to expertise. However, Chassin and Loeb found that the methods that high-reliability organizations currently use cannot be directly applied to healthcare organizations. Instead, they suggested that a series of incremental changes must occur based on leadership's commitment to achieve zero harm to the patient, a fully functional culture safety inculcated throughout the organization, and widespread deployment of highly effective process tools.

Leadership commitment to zero patient harm by hospital boards and CEOs with physicians routinely directing clinical quality improvement activities is critical. If this commitment is not the number one priority and has to compete with other priorities, it is difficult to imagine that high reliability will emerge as a resolution. This concept is supported by studies reporting on negative safety cultures and patient injuries, deaths, and medical errors (Institute of Medicine, 2004; Muething et al., 2012; Ormsby, 2013; McFadden, Stock, \& Gowen III, 2014; Taylor, Dominici, Agnew, Gerwin, Morlock, \& Miller; 2012), with Ormsby (2013) further noting that alignment of patient and worker safety improvement initiatives is necessary for optimal outcomes, efficiencies, and the development of organizational cultures of safety. The research model compiled by McFadden et al. (2014) from 204 hospitals and supplemented with objective outcome data from the Center for Medicare and Medicaid (CMS) in particular provided empirical evidence that a safety climate connected to the CEO's transformational leadership style was related to continuous quality improvement (CQI) and improved process quality. However, the authors also noted that CQI initiatives are also related to higher hospital-acquired condition rates, a patient safety measurement. While the safety climate is correlated to improved patient safety outcomes, most importantly, the authors concluded that patient safety climate and CQI initiatives are not interchangeable or universally beneficial. This is a really crucial point; CQI does not lead to an improved patient safety climate alone.

Chassin and Loeb also discuss the vital requirement that health information technology (IT) must be coordinated and integrated. Results of leading hospitals implementing comprehensive electronic health record (EHR) systems have been positive because of strong leadership, full involvement of clinical staff in design and implementation, mandatory staff training, and strict adherence to timelines and budgets (Silow-Carroll, Edwards, \& 
Rodin, 2012). But most of all, successes were achieved because of coordination and integration. In contrast, when this does not happen, the result is a colossal failure, as has occurred in Veterans Affairs (VA) Hospitals (Buckley, 2013). Additionally, IT technologies must be easy to use and useful in terms of improving job performance, efficiency, and/or quality (Karsh, 2004). Karsh also indicates that such technology must be used in the manner intended and provide satisfaction by users, which can be achieved via pilot testing at selected locations with a person responsible and accountable for success of the implementation. Mandl and Kohane (2012) also suggest that one of the reasons most end users have had such a hard time is that commercially available EHR systems lack flexibility, interoperability, and easy-to-use tools; are vastly overpriced; and are poorly supported in both initial installations and ongoing change.

The development of a fully functioning safety culture occurs in stages, and includes five important aspects: trust, accountability, identifying unsafe conditions, strengthening systems, and assessment (Chassin \& Loeb, 2013). Although far from being aligned with these aspects, the most current AHRQ assessment indicates that teamwork within units (a measure of trust), supervisor/manager expectations and action promoting patient safety (elements of unsafe conditions, strengthening systems, and assessment), and organizational learning (part of unsafe conditions) have all improved, with 81, 76, and 73\% of responses positive (Agency for Healthcare Research and Quality, 2014). This is an improvement but Chassin and Loeb's detailed assessments show there is still a long way to go. However, the AHRQ's nonpunitive response to error measure, which relates to the accountability element, shows that $56 \%$ of responses are negative; this indicates that accountability is still likely to be a major issue in most U.S. hospitals.

Strength and resilience of safety systems is another factor listed by Chassin and Loeb. Currently, most hospitals react to medical errors - conducting root cause analyses and instituting corrective action to prevent such occurrences. However, hospitals need to move to a state of proactive assessment, constantly examining current safety systems and reducing risk, and allowing reporting of adverse events without sanctions against participants who might not otherwise report such events. Integrated assessment of adverse events is important so that weaknesses in systems can be identified. Illustrative of the current situation, the state of Minnesota requires that hospitals and ambulatory surgical centers (ASCs) submit adverse event reports to the state health department, which compiles events into an annual report and recommendations for improvement (DeJohn, 2013). Within 15 days, each adverse event must be reported followed by development of an action plan that the facility has to assess within a further 3090 days in terms of its success. This entire process can be seen as reactive (and forced upon the participating entities) rather than proactive at the level of the hospital or ASC. Obviously, this is the first stage of a long series of changes, but at some point the transition to entity-based, strong, resilient safety systems must begin.

Last, robust improvement must be initiated. Chassin and Loeb identify three elements in this process: (1) lean: tools and a philosophy based on employee-empowered improvement to identify and remove wasted effort without compromising the quality of outcomes; (2) six sigma: tools for continually improving process outcomes that will dramatically reduce the frequency of defective products or outcomes; and (3) change management, a process that prepares organizations to accept, implement, and sustain the application of lean and six sigma tools that will improve processes - not just a case of paying "lip service." At present, most hospitals and healthcare organizations do not identify with other industries, which have used these tools for many decades to develop best practices and improve their operations. This must change; using the excuse that healthcare organizations are special exempt cases is a fallacy.

Healthcare organization worker fatigue also has been identified as an issue that could be linked to patient outcomes and in 2003 the Accreditation Council for Graduate Medical Education (ACGME) mandated that all US residency programs limit work hours for trainees (Accreditation Council for Graduate Medical Education, 2003). Only a few studies have examined whether these changes helped patient outcomes. One example is the study of Salim et al. (2007), who compared death and complication rates for adult trauma patients admitted before the changes (an 80-hour work week) during 2001-2003 versus rates after the changes (2004-2006) at an academic level 1 trauma center. Surprisingly, while there was no change in fatalities, there was a statistically significant increase in total, preventable, and nonpreventable complications that was unexplained. At the behest of Congress, the IOM embarked on a year-long examination of the evidence available regarding physician sleep, clinical performance deficits, and medical errors. The report recommended new limits on resident duty hours and workload, increased supervision, more focus on resident physician safety, and training in structured handovers and quality improvement, 
amongst other things (Institute of Medicine, 2008). While the ACGME issued new rules that went into effect on July 1,2011 , they fell far short of the IOM's recommendations, including a restriction that the maximum shift length of 16 hours only be applied to first-year residents. In response to the new ACGME rules, a comprehensive paper whose focus was implementation of the IOM's recommendations was published in the same year (Blum, Shea, Czeisler, Landigran, \& Leape, 2011). While the recommendations of Blum et al. (2011) are lengthy, there are a few extremely salient points:

- $\quad$ Adjust resident physician workload requirement to optimize educational value

- $\quad$ Train both resident and attending physicians in effective teamwork

- $\quad$ Always have good, supervising physicians for residents assigned to critically ill patients

- $\quad$ Limit resident physician work hours to 12-16 hours per shift with a minimum of 10 hours off duty between shifts

- $\quad$ Hospitals should adhere to principles of sleep and circadian science in regard to schedules

- $\quad$ Training in fatigue recognition for residents with assistance by the hospital for transportation to and from home if needed

- More effective training in handing over of care, in a team setting if possible, and integration of handovers into EHRs

- $\quad$ External oversight of ACGME by CMS and the Joint Commission

It is not known with any certainty if the newest rules are affecting patient safety yet. While most of the ACGME rule changes affected residents, it is also clear that surgeons operating in the United States also have problems. Authors of a cross-sectional survey of 7,905 surgeons in 2008 using standardized instruments for burnout and quality of life (QOL) found that $9 \%$ of surgeons had made a major medical error in the past 3 months, with 70\% of these surgeons indicating it was attributable to individual factors (Shanafelt et al., 2009). Commitment of medical errors was strongly associated with mental QOL, especially depression and burnout. While it is known that stress and fatigue are associated with medical errors, this is one area that needs much more focus in the future.

Other factors identified in a study between 2000 and 2004 utilizing MEDMARX, an anonymous, confidential, de-identified, Internet-accessible medication error-reporting program, included ER errors that occurred in the administrative phase of the medication process with peak error rates on Mondays and during the evening shift (Pham et al., 2011). The authors noted common factors of distraction, emergency situations, and workload increases. Ultimately, understaffing, inexperienced staff, and overcrowding contribute heavily as causes.

\section{STRATEGIES AND TOOLS THAT COULD HELP FURTHER IMPROVEMENT}

A recent national (U.S.) estimate of the direct costs associated with medical errors at hospitals suggests it was over $\$ 1$ billion in 2009 , but this is likely a considerable underestimate as repeat injuries, certain kinds of injury, visits associated with excessively high costs $(>\$ 300,000)$, costs associated with follow-up visits, and litigations costs were excluded (David, Gunnarsson, Waters, Horblyuk, \& Kaplan, 2013). Thus, the true costs of medical errors could be vastly more than estimated.

Another reason why the costs of medical errors remain uncertain is that they are under-reported. Perez et al. (2014) suggest that in part this is because of intrapersonal, interpersonal, institutional, and societal barriers to transparency and error disclosure. Most importantly, physicians face recriminations from "patients, lawyers, hospital-employers, insurance companies, and their own conscience, along with the threats of incurring legal and financial penalties" (Perez et al., 2014, p.49). Thus, to obtain better estimates of reporting, the "blame" culture embedded in many healthcare facilities has to be modified, and hospital workers must be willing to step up to the plate and report when something goes awry, even if it is not obvious that a medical error has occurred.

There is no doubt that in the right hands the Global Trigger Tool can help the medical community to better quantify errors and identify sources of error. For example, the Adventist Health system in a review of 17,295 patient records found that adverse events clustered as medication-related glycemic events; medication-related delirium; confusion, or oversedation related to analgesics, sedatives, and muscle relaxants; pressure ulcers; medication-related 
bleeding; and medication-related skin/mucosal reaction/itching (Garrett Jr., et al., 2013). This enabled Adventist to focus on systemwide collaborative projects to improve glycemic and pressure ulcer management.

The initiative conducted by Muething et al. (2012) at the Cincinnati Children's Hospital Medical Center is illustrative as it built on three strategic plans confirming quality as a core business strategy since 2001. The multidisciplinary team-based 2006 safety initiative supported by hospital leaders focused on error prevention; restructuring safety governance; a new root cause analysis process and a common cause database; and specific tactical interventions for high-risk areas. Serious safety events were reduced by two thirds and after encountered difficulties in the first year, patient safety culture outcomes improved between 2007 and 2009.

Computer provider order entry (CPOE) systems were originally promoted by the IOM as a means of reducing medication errors and patient harm (Committee on Quality of Health Care in America, 2001). Researchers recently estimated the effect of CPOE on medication errors using random effects meta-analysis in a systematic review context and combined the results with data from the 2006 American Society of Health-System Pharmacists Annual Survey, and the society's 2008 Electronic Health Record Adoption Database (Radley, Wasserman, Olsho, Shoemaker, Spranca, \& Bradshaw, 2013). The results indicated that CPOE systems could reduce the likelihood of prescription errors by $48 \%$ with a $12.5 \%$ reduction of medical errors in 2008 , translating to a national 17.4 million medication errors averted.

Placing orders in the wrong patient's electronic medical record is another factor causing medical errors, and was found in a recent investigation to be the second most common cause of care being provided to the wrong patient, responsible for 24\% of reported errors (Hyman, Laire, Redmond, \& Kaplan, 2012). Fifteen months after instituting an order verification screen with a patient's photo centrally placed within the screen, no unintended care errors resulted when the photo was present. This simple strategy appears to be highly effective.

Finally, in the era of Universal Protocol introduced by the Joint Commission in 2004, a study was conducted to determine the frequency, root causes, and outcomes of wrong site and wrong patient procedures (Stahel et al., 2010). During 2002 through 2008, 25 wrong-patient and 107 wrong-site procedures were identified out of 27,370 physician self-reported adverse occurrences. In $20 \%$ and $36 \%$, respectively, of these procedures, significant patient harm occurred. Errors in diagnosis and errors in communication were responsible for wrongpatient procedures while errors in judgment and lack of performing a "time-out" (a moment prior to surgery in the operating room in which staff checks that everything is in order, including right patient, right site, and right surgery) were the primary causes of wrong-site procedures. While at some health care facilities implementation of the Universal Protocol clearly needs improvement, the protocol itself has been judged flawed because "it fails to account for the complexities of the surgical process and all the different ways in which risks of a wrong-site procedure may be introduced into it" (Chassin and Loeb, 2013, p.463).

\section{CONCLUSIONS}

In the last 15 years progress in managing medical errors has been limited. Part of the problem emanates from the nature of the systems used to identify such errors. However, newer systems seem to fair better in this respect. While there are bright spots in particular healthcare facilities within the United States, the changes needed to make healthcare systems into high-reliability organizations have a long way to go. Moreover, IT systems for healthcare organizations are far more expensive than they should be and frequently lack integration. Bowman (2013) elegantly captured some of the issues with electronic health records by stating "Designed and used inappropriately, EHRs add a layer of complexity to the already complex delivery of healthcare, leading to unintended adverse consequences such as dosing errors, failure to detect serious illnesses, and delays in treatment due to poor humancomputer interactions or loss of data" (p.9). In summary, therefore, healthcare organizational leaders are just beginning to face a long series of perhaps painful transitions that will be needed to arrive at the level of most industrial organizations in respect to safety. 


\section{AUTHOR INFORMATION}

Dr. Yvette Ghormley holds several degrees, including a $\mathrm{PhD}$ in Organization and Management with a concentration in E-Commerce, an MA in Education and Human Development with a concentration in Education, Technology and Leadership, and an MA in Information Technology, Information Security. In addition, she holds graduate certificates in Acquisition and Contract Management and Project Management. Dr. Ghormley has presented at numerous conferences and has published in International journals, texts, and handbooks. She is well known for using technological initiatives in business and entrepreneurship for non-profit and for-profit enterprises.

\section{REFERENCES}

Agency for Healthcare Research and Quality. (2014). 2014 user comparative database report. Hospital survey on patient safety culture. Retrieved December 17, 2014, from http://www.ahrq.gov/professionals/qualitypatient-safety/patientsafetyculture/hospital/2014/index.html

Accreditation Council for Graduate Medical Education (2003). Resident duty hours in the learning and working environment. Comparison of 2003 and 2011 standards. Retrieved December 15, 2014, from https://www.acgme.org/acgmeweb/Portals/0/PDFs/dh-ComparisonTable2003v2011.pdf

Bleich, S. (2005). Medical errors: five years after the IOM report. Issue Brief (Commonwealth Fund), 830, 1-15.

Blum, A. B., Shea, S., Czeisler, C. A., Landrigan, C. P., \& Leape, L. (2011). Implementing the 2009 Institute of Medicine recommendations on resident physician work hours, supervision, and safety. Nature and Science of Sleep, 3, 47-85.

Bowman, S. (2013). Impact of electronic health record systems on information integrity: Quality and safety implications. Perspectives in Health Information Management, 10, 1c (e collection).

Buckley, K. (2013). VA electronic health records: What federally mandated health integration really looks like. National Center for Policy Analysis Issue Briefs, No. 120. Retrieved December 16, 2014, from: http://www.ncpa.org/pub/ib120

Chassin, M., \& Loeb, J. M. (2013). High-reliability health care: getting there from here. The Milbank Quarterly, 91(3), 459-490.

Classen, D. C., Resar, R., Griffen, F., Federico, F., Frankel, F., Kimmel, N., et al. (2011). 'Global Trigger Tool' shows that adverse events in hospitals may be ten times greater than previously measured. Health Affairs (Millwood), 30(4), 1-9.

Committee on Quality of Health Care in America, Institute of Medicine. Kohn, L., Corrigan, J., \& Donaldson, M. (Eds.). (1999). To err is human: Building a safer health system. Washington, DC: National Academy Press.

Committee on Quality of Health Care in America, Institute of Medicine. (2001). Crossing the quality chasms: a new health system for the $21^{\text {st }}$ century. Washington, DC: National Academy Press.

David, G., Gunnarsson, C. L., Waters, H. C., Horblyuk, R., \& Kaplan, H. S. (2013). Economic measurement of medical errors using a hospital claims database. Value in Health, 16(2), 305-310.

DeJohn, P. (2013). Minnesota's adverse event reporting system has led to patient safety improvements. $O R$ Manager, 28(6), 1-2.

Department of Health and Human Services, Office of Inspector General. (2010). Adverse events in hospitals: National incidence among Medicare beneficiaries. No. OEI-06-09-00090. Washington DC: HHS.

Garrett Jr., P. R., Sammer, C., Nelson, A., Paisley, K.A., Jones, C., Shapiro, E., et al. (2013). Developing and implementing a standardized process for Global Trigger Tool application across a large health system. The Joint Commission Journal on Quality and Patient Safety, 39(7), 292-297.

Hyman, D., Laire, M., Redmond, D., \& Kaplan, D. W. (2012). The use of patient pictures and verification screens to reduce computerized provider order entry errors. Pediatrics, 130(1), e211-e219.

Institute for Healthcare Improvement. (2009). Introduction to trigger tools for identifying adverse events. Cambridge, MA: IHI.

Institute of Medicine. (2004). Keeping patients safe: transforming work environment of nurses. Retrieved December 16, 2014, from http://www.nap.edu/catalog/10851/keeping-patients-safe-transforming-the-workenvironment-of-nurses

Institute of Medicine. (2008). Resident duty hours: Enhancing sleep, supervision and safety. Washington, DC: National Academies Press. 
James, J. T. (2013). A new, evidence-based estimate of patient harms associated with hospital care. Journal of Patient Safety, 9(3), 122-128.

Karsh, B. T. (2004). Beyond usability: designing effective technology implementation systems to promote patient safety. Quality and Safety in Health Care, 13(5), 338-394.

Mandl, K. D. \& Kohane, I. S. (2012). Escaping the EHR trap-the future of heath IT. New England Journal of Medicine, 366(24), 2240-2242.

McFadden, K. L., Stock, G. N., \& Gowen III, C. R. (2014). Leadership, safety climate, and continuous quality improvement: Impact on process quality and patient safety. Health Care Management Review, 40(1), 2434.

Ormsby, D. J. (2013). Integrating patient and worker safety policies. New Solutions, 23(2), 315-325.

Perez, B., Knych, S. A., Weaver, S. J., Liberman, A., Abel, E. M., Oetjen, D. et al. (2014). Understanding the barriers to physician error reporting and disclosure: A systemic approach to a systemic problem. Journal of Patient Safety, 10(1), 45-50.

Pham, J. C., Story, J. L., Hicks, R. W., Shore, A. D., Morlock, L. L., Cheung, D. S., et al. (2011). National study on the frequency, types, causes, and consequences of voluntarily reported emergency department medication errors. The Journal of Emergency Medicine, 40(5), 485-492.

Radley, D. C., Wasserman, M. R., Olsho, L. E., Shoemaker, S. J., Spranca, M. D., \& Bradshaw, B. (2013). Reduction of medication errors in hospitals due to adoption of computerized provider order entry systems. Journal of the American Medical Informatics Association, 20(3), 470-476.

Reason, J. (1997). Managing the risks of organizational accidents. Burlington, VT: Ashgate.

Shanafelt, T. D., Balch, C. M., Bechamps, G., Russell, T., \& Dyrbye, L. et al. (2009). Burnout and medical errors among American surgeons. Annals of Surgery, 251(6), 995-1000.

Silow-Carroll, S., Edwards, J. N., \& Rodin, D. (2012). Using electronic health records to improve quality and efficiency: the experience of leading hospitals. Issue Brief (Commonwealth Fund), 17, 1-40.

Stahel, P. F., Sabel, A. L., Victoroff, M. S., Varnell, J., Lemibitz, A., Boyle, D. J., et al. (2010). Wrong-site and wrong-patient procedures in the Universal Protocol era. Analysis of a prospective database of physician self-reported occurrences. Archives of Surgery, 145(10), 978-984.

Taylor J. A., Dominici, F., Agnew, J., Gerwin, D., Morlock, L., \& Miller, M. R. (2012). Do nurse and patient injuries share common antecedents? BMJ Quality and Safety, 21(2), 101-111.

Weick, K. E., \& Sutcliffe, K. M. (2007). Managing the unexpected. $2^{\text {nd }}$ ed. San Francisco: Jossey-Bass. 
NOTES 